\title{
Clinical definitions and classification of cardiomyopathies
}

\author{
C. M. OAKLeY
}

CARDIOMYOPATHY is simply heart muscle disease, but it is surprisingly difficult to find a common language whereby we know that we are talking about the same thing. The study and recognition of cardiomyopathies has been delayed because of the semantic difficulties which we have encountered. The first working classification to be generally adopted (Goodwin et al., 1961) was a clinical classification into congestive, constrictive and hypertrophic obstructive types. This clinical classification antedated our present understanding of the haemodynamic derangements and it lacks pathological specificity. Congestive cardiomyopathy simply means that the patient has left ventricular pump failure which may result from many different pathologies involving the left ventricle. We need something new if we are not to struggle with three concurrent classifications, one clinical, one functional and one pathological.
Although many different disease processes can give rise to left ventricular dysfunction, in the vast majority of patients with heart muscle disease no specific aetiology can be determined by either clinician or pathologist. Before deciding that a patient has a primary cardiomyopathy it is necessary to consider carefully and to exclude all recognized or possible causes of the myocardial problem.

From the viewpoint of haemodynamic function we can recognize the difference between a ventricle which is dilated because it fails to empty at each beat and one which is hypertrophied and not dilated but is stiff during diastolic filling. Turning to aetiology, we can classify cardiomyopathies according to whether there is a known cause or whether there is a disease elsewhere in the body. In view of the magnitude of the problem presented by the common heart failures of unknown origin it appears important

TABLE 1. Aetiological classification of heart muscle disorder

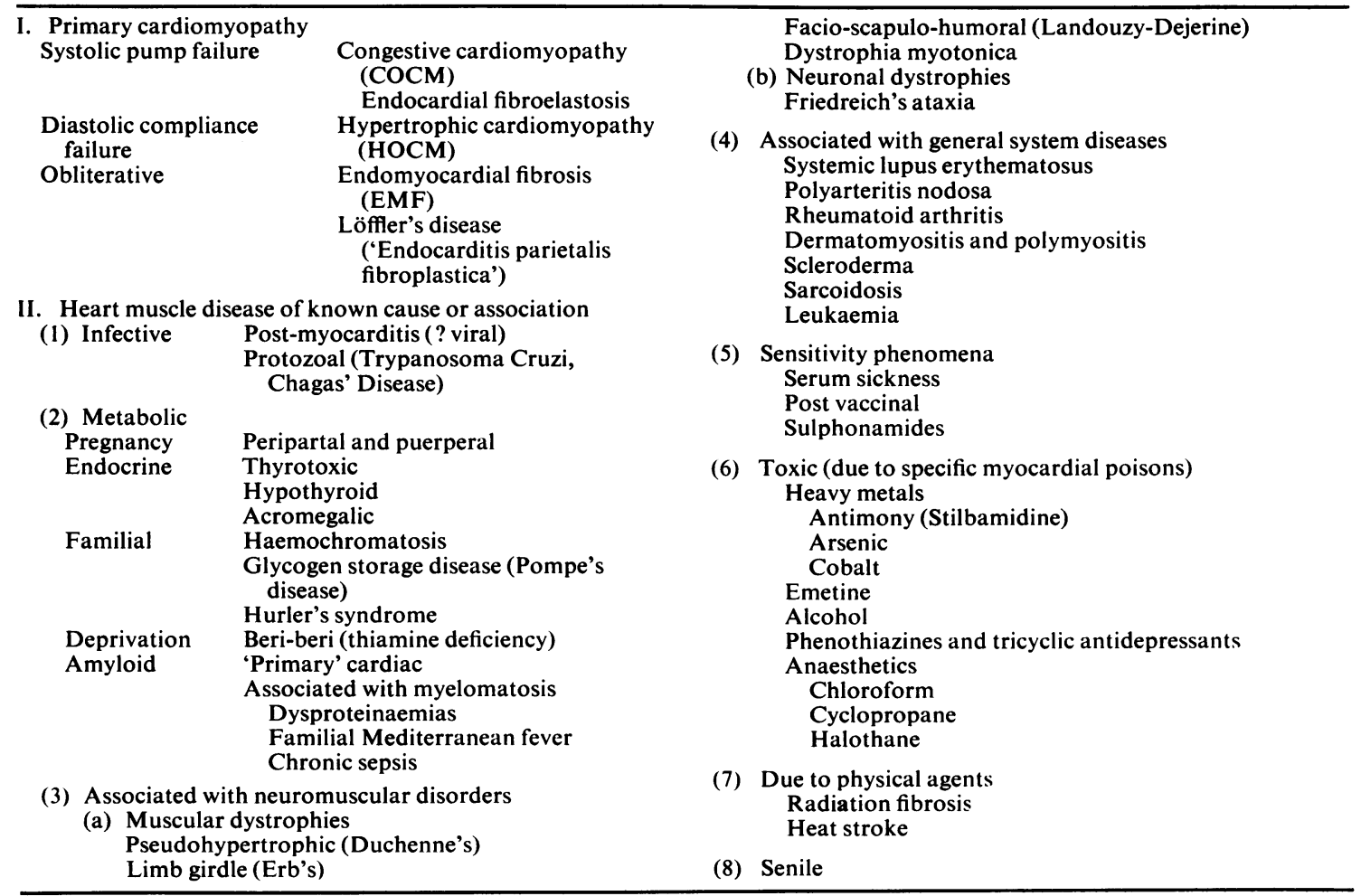


to polarize our research endeavours and advise that the term cardiomyopathy should be reserved for 'heart muscle disorder of unknown cause or association' and that heart muscle disease of known cause or association should be so described, for example, sarcoid heart disease, amyloid heart disease, etc. If we accept this concept, we can narrow the problem down and concentrate first on functional differences between the cardiomyopathies.

There is the primary pump failure with a dilated cavity (congestive cardiomyopathy) with compensatory hypertrophy. The cause of the hypertrophy is clear. Then there is hypertrophic cardiomyopathy where the cause of the hypertrophy is not clear and may at this stage of our knowledge be considered to be the primary event in this condition. The cavity is of normal size but may be of unusual shape. In this hypertrophic cardiomyopathy systolic pump failure occurs late or not at all, the problem being a primary diastolic compliance failure associated with the abnormal and hypertrophied muscle. There also exists a third group, the obliterative cardiomyopathies. They are endomyocardial fibrosis (EMF) and Löffler's disease which may perhaps be the same conditions described respectively from tropical areas and from temperate zones. They should not really be included with the cardiomyopathies because there is no evidence that the myocardium itself is primarily involved. In these conditions, atrio-ventricular valve immobilization is associated with progressive obliteration of the ventricular cavities, either side of the heart being affected in EMF but predominantly the left ventricle in Löffler's disease.

Here we have a functional classification for the primary cardiomyopathies (Fig. 1) which has pathological counterparts. If instead of 'congestive' we think of systolic pump failure, and if instead of 'hypertrophic' we think of diastolic compliance failure we are making progress; we now have a clinical and haemodynamic classification with a pathological counterpart. All the secondary heart muscle diseases of known cause or association produce systolic pump failure. Any insult to the myocardium if it is sufficiently severe, will result in inability of the left ventricle to empty and secondary dilatation.

Hypertrophic (obstructive) cardiomyopathy or HOCM, is a group of conditions which is very likely of homogeneous origin and genetically determined even in those without clear familial incidence. It exists either with obstruction to outflow, which was how it was originally recognized, or without any obstruction to outflow, which has only more recently been recognized. Rarely, patients with obstruction spontaneously lose their obstruction and sometimes obstruction has never been present at all, so that we now recognize hypertrophic cardiomyopathy essen-
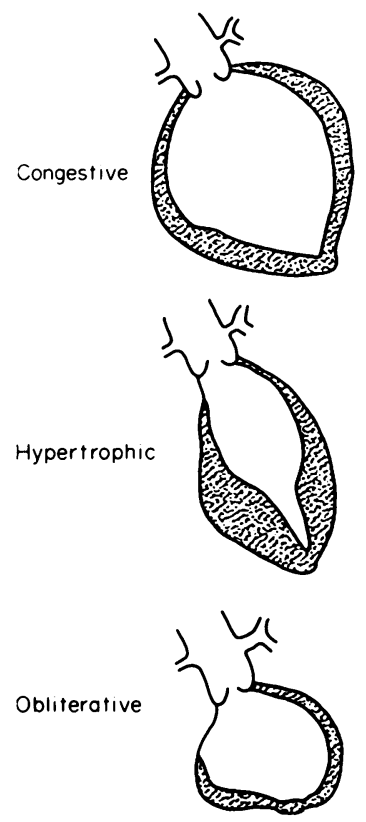

Fig. 1. Classification of cardiomyopathy. 'Congestive' refers to systolic pump failure; 'hypertrophic' refers to diastolic compliance failure; 'obliterative' describes endomyocardial fibrosis and Löffler's disease.

tially by the demonstration of hypertrophy without a cause. I am now going to discuss only these two conditions, pump failure (COCM) and compliance failure (HOCM).

In congestive cardiomyopathy or COCM (Fig. 2) there is failure of the left ventricle to empty due to loss of contractile force. The heart is usually quite large but the shorter the history, the smaller the heart tends to be. Conversely, when the condition has gone on a long time and achieved a measure of compensation there is often very considerable secondary hypertrophy. In examining the factors which determine longevity in COCM, Dr Richard Croxton and colleagues at Hammersmith Hospital looked at the relationship between left ventricular muscle mass and the degree of cavity enlargement (Fig. 3). When analysed according to whether the patient had survived or died it was found that the patients who had died showed relatively less hypertrophy (with a high cavity-width to wall-thickness ratio) than the patients who had survived, who had the same cavity-to-wall thickness ratio as the normal subject. In other words, the survivors seemed to have succeeded in compensating by hypertrophy for the cavity dilatation and the increase in wall tensions which resulted from it. So the hypertrophy in COCM appears to be beneficial. 


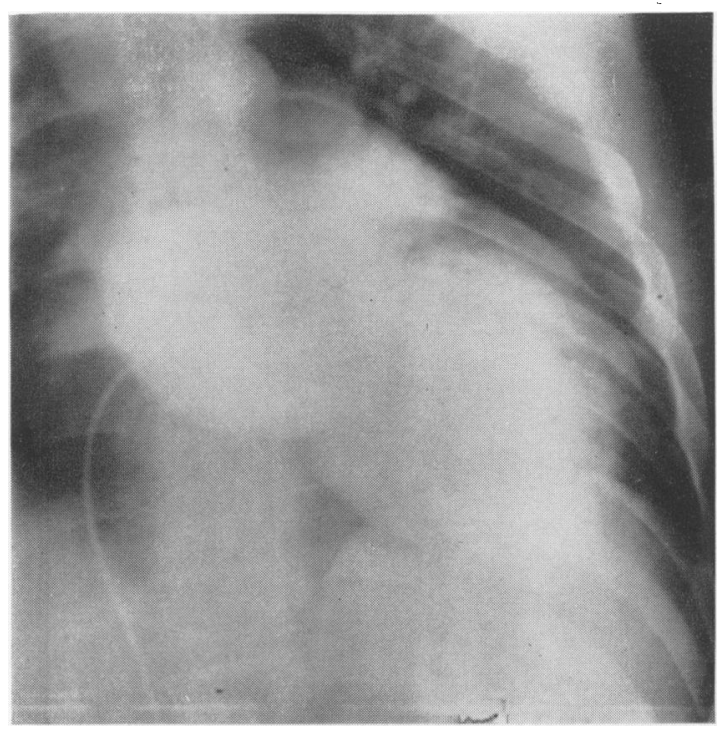

Fig. 2. The appearance of the left atrium and ventricle in COCM. These chambers are grossly dilated.

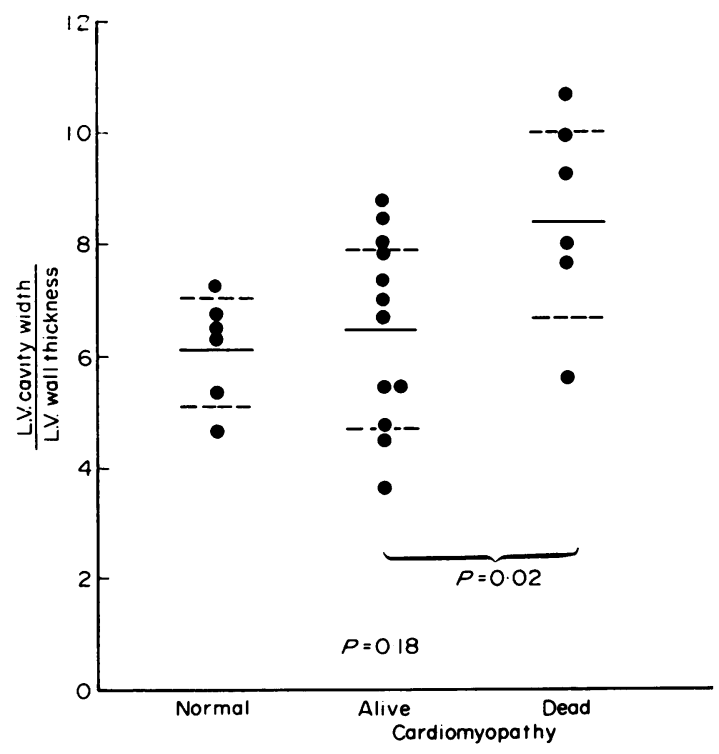

Fig. 3. Diagram to illustrate the better prognosis of COCM when there is adequate relative hypertrophy.

Some of these patients when advanced in their disease may have angina and some may have an infarct pattern on the ECG. Selective coronary angiograms in fourteen patients in whom the diagnosis of a cardiomyopathy was considered revealed that in this country at least one cannot be absolutely certain that a patient with left ventricular failure is not suffering from coronary artery disease until selective coronary angiography has been done. This does not mean that selective coronary angiography should necessarily always be carried out in such patients. Left ventricular failure does not follow a single major coronary stenosis or occlusion. Patients only develop frank failure after two or more major occlusive lesions or after the development of a localized ventricular aneurysm. Of course, patients with COCM are not immune from coronary atheroma although as a group they seem to be singularly free from it.

The differential diagnosis of COCM therefore includes:

(1) ischaemic heart disease;

(2) hypertensive heart failure (to which we shall refer later);

(3) chronic pericardial effusion (which is recognized by a normal ECG and no gallop sounds);

(4) severe right heart failure resulting from conditions such as obliterative pulmonary hypertension;

(5) aortic stenosis in severe low output failure when the murmur may virtually disappear (important but rare).

When the heart is less enlarged in congestive cardiomyopathy, a different set of diagnostic possibilities present themselves. These are usually patients with a shorter history and the differential diagnoses are less common conditions. When a patient presents with the clinical syndrome of congestive heart failure, one dare not assume that the left ventricle is dilated; patients with HOCM who have no systolic pump failure may develop such a high left ventricular filling pressure from the increased inflow resistance that congestive heart failure supervenes in the absence of left ventricular cavity dilatation. Such patients may only be recognized after left ventricular angiography; Fig. 4a shows such a case, and for comparison 'typical' HOCM with obstruction (Fig. 4b). The normal person ejects about two-thirds of the left ventricular contents at each beat. Patients with COCM eject much less than that and patients with HOCM generally eject at least the normal amount and sometimes seem to empty their cavity more than in the normal, but in an advanced state they may empty less well, still without dilating. The result is a profound fall in stroke output.

The obstruction to outflow from the left ventricle which occurs in some, but not all, patients with HOCM seems to be due to an excess of hypertrophied muscle in the septum at a point in the left ventricular outflow tract where there is normally not very much space; the obstruction occurs where the anterior cusp of the mitral valve hits the septum during systolic ejection. This may occur because of 

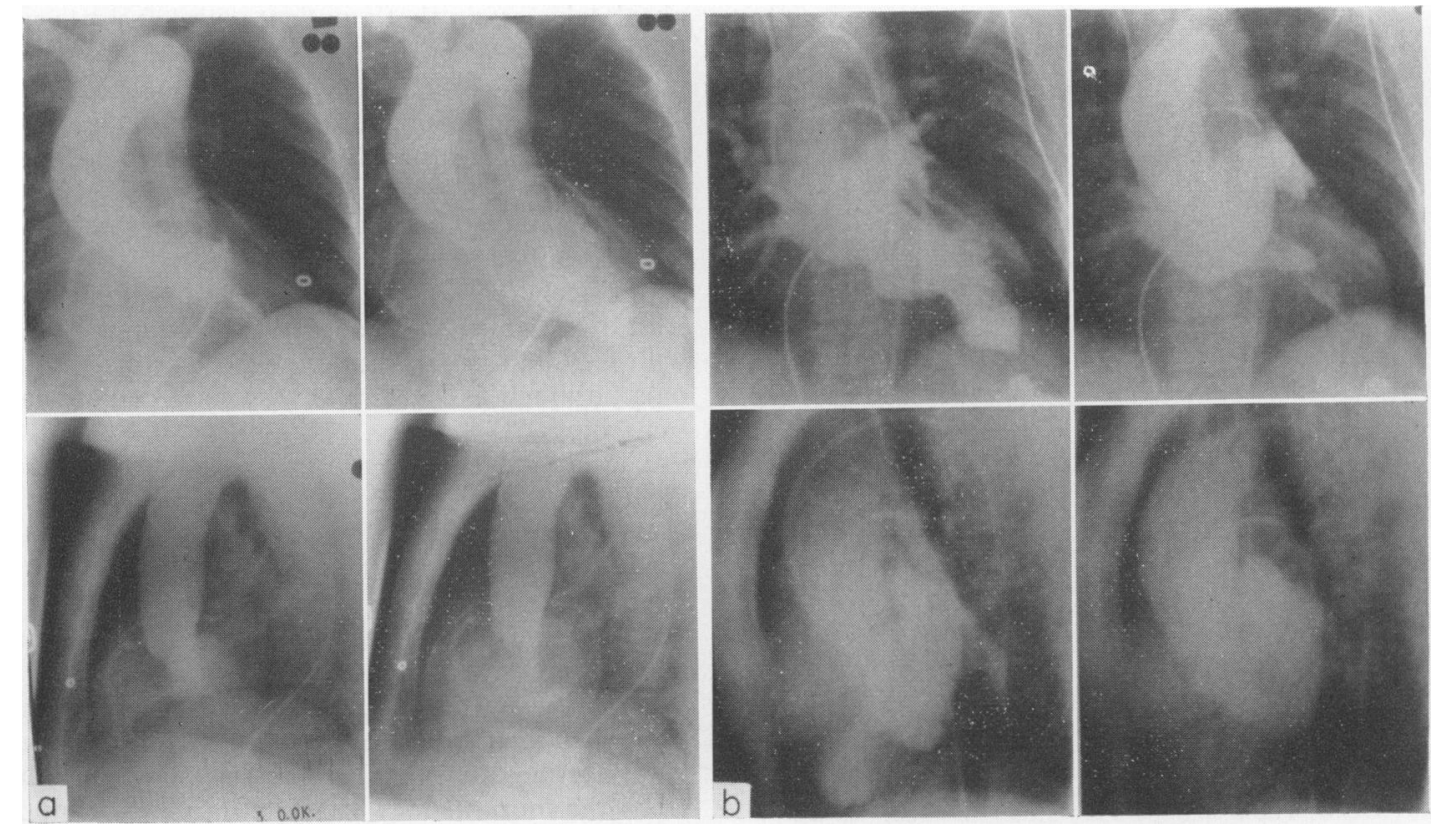

FIG. 4. (a) Retrograde LV angiogram in HOCM without obstruction. Massive generalized hypertrophy is obvious but apart from increased papillary muscle indentations the shape of the cavity is not greatly distorted in systole (top left). The cavity is of normal size. There is no mitral reflux (bottom left). Apart from the hypertrophy the appearance in diastole is normal. The appearance of the LV angiogram does not differ between those with and those without clinical congestive failure.

(b) Transseptal left atrial angiogram in HOCM with obstruction. Note the distortion of the cavity with gross septal bulge. The cavity is almost bereft of contrast medium at the completion of systole.

compensatory over-contraction of a relatively uninvolved free-wall musculature. Other patients with gross left ventricular hypertrophy may have no outflow obstruction. This seems to be almost accidentally determined by the site of the hypertrophied muscle, the unobstructed patients having diffuse rather than local septal dystrophic muscle. Angiograms in HOCM show gross hypertrophy, and very often a bent cavity shape in those with obstruction: sometimes one can see the site of the latter (Fig. 5). This lateral LV angiogram from a patient with HOCM and outflow-tract obstruction shows the aortic valve cusps to be wide open during full systolic ejection but the anterior cusp of the mitral valve is open in the diastolic position and hitting the septum. This explains the narrowing of the orifice during ejection and also explains the development of late systolic mitral reflux. The thin mitral cusp moves very fast and is difficult to see on angiograms; indeed it is frequently invisible unless the plane or projection is perfectly correct. This illustration also shows how the anterior cusp by moving forward allows mitral reflux to occur. This produces the characteristic murmurs which are late in onset because early ejection is unimpeded but have an explosive onset coincident with dislocation of the mitral valve and are decrescendo from there on in typical cases (Fig. 6). An atrial teat is nearly always

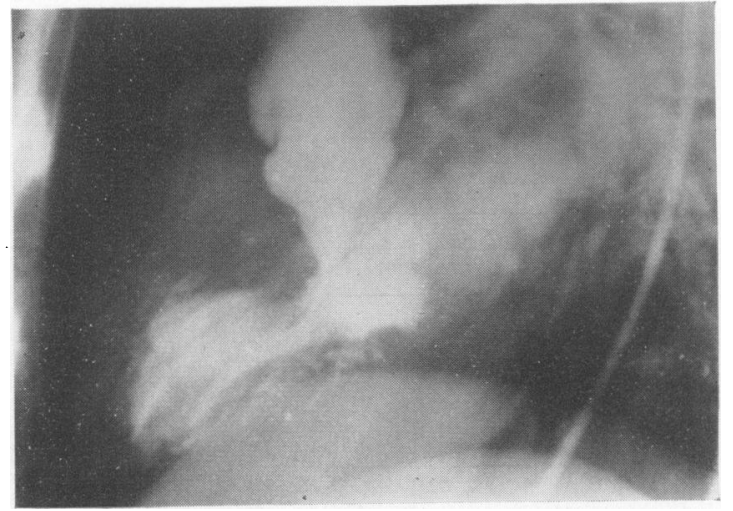

FIG. 5. Left lateral LV angiogram showing the anterior dislocation of the anterior mitral cusp and mitral reflux during mid-ejection. 


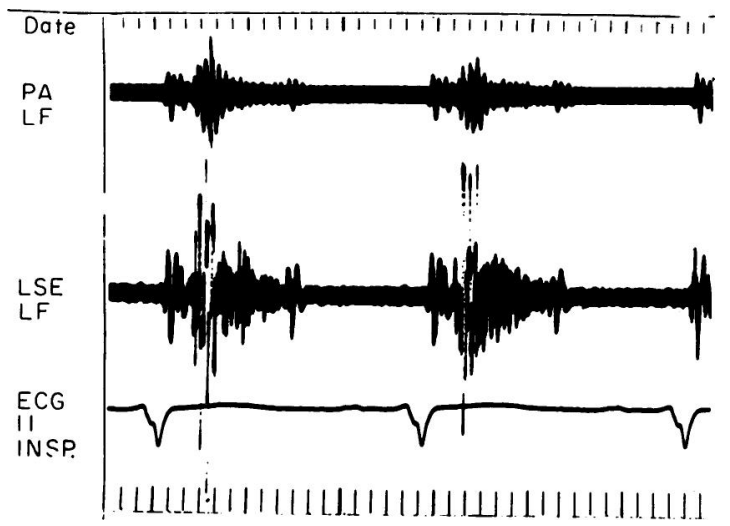

FIG. 6. Phonocardiogram from HOCM with obstruction.

The explosive onset of the systolic murmur is well shown.

palpable at the apex and indicates a raised enddiastolic pressure within the left ventricular cavity, a most important physical sign. Sometimes there is also a third heart sound in patients with obstruction and mitral reflux but the atrial beat is the hallmark of this disorder and is nearly always present.

The obstruction to outflow can be shown on a withdrawal trace from the left ventricle (Fig. 7). The subvalvar region of the left ventricle above the anterior cusp of the mitral valve shows the same systolic pressure as in the aortic root. Notice the characteristic systolic dip in the arterial pulse which follows the onset of obstruction to outflow and at the same time the onset of mitral regurgitation. This systolic dip is not specific but it is characteristic of this disorder; it gave rise to an erroneous idea that these ventricles are superventricles, with enhanced force and hyperkinetic, which they are not. The apparent hyperkinesia is due to the systolic dip in the pressure which gives the pulse its jerky character. Early on in our experience we were all obsessed by this obstruction to outflow. It is immensely labile, so that any manoeuvre or drug which alters left ventricular cavity size will also alter the amount of obstruction. Amylnitrite, a drug with no direct effect on the myocardium, by lowering central blood volume greatly augments obstruction and with its development, the systolic dip in the arterial pulse appears. When the obstruction is removed by giving phenylephrine, a pure vasoconstrictor which distends the left ventricle in systole, the obstruction disappears ard the arterial pulse assumes a normal contour. HOCM patients without obstruction to outflow have either perfectly normal or small volume arterial pulses. It is the high left ventricular enddiastolic pressure (LVEDP) which produces the symptoms (Fig. 8); left atrial and pulmonary capillary hypertension make the patient breathless. The height of the LVEDP reflects the amount of hypertrophy and fibrosis in the ventricle and the resulting impairment of filling eventually brings about death.

The electrocardiogram can be very variable in HOCM but one of the common appearances is shown in Fig. 9a, with florid left ventricular voltage increase and repolarization changes. The absence of a septal $Q$ wave in the left ventricular leads and sometimes a short PR interval resemble a WolffParkinson-White or pre-excitation syndrome but without delta wave. These patients rarely get paroxysmal tachycardia and His bundle studies suggest that this is not true pre-excitation (also see $9 b$ and $9 c)$.

The chest X-ray can also vary considerably but the heart is rarely very large, the aorta is of normal size, there is no valve calcification and the left ventricle often looks 'squared off' (Fig. 10). The left atrium may be enlarged and there may be evidence of a high left atrial pressure, in which case the question of mitral stenosis may be raised.

The differential diagnosis of hypertrophic cardiomyopathy with obstruction is that of other conditions which give rise to systolic murmurs. Once you start to wonder which of the five main differential diagnoses the patient has, then he almost certainly has HOCM! In aortic stenosis, the carotid pulse should be slow and sustained, there should be an ejection click if the patient is young, or a soft aortic closure

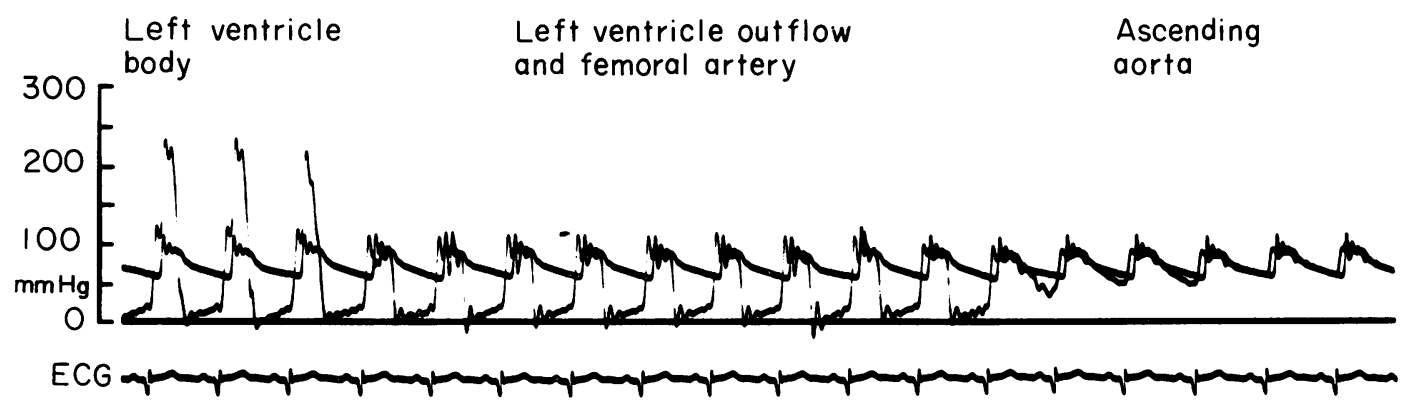

Fig. 7. Withdrawal of catheter from left ventricle with femoral artery trace showing the subvalvar site of the obstruction. 

E.C.
Control
Amyl nitrite
Phenylephrine
H.R. 60
H.R. 70
H.R. 40

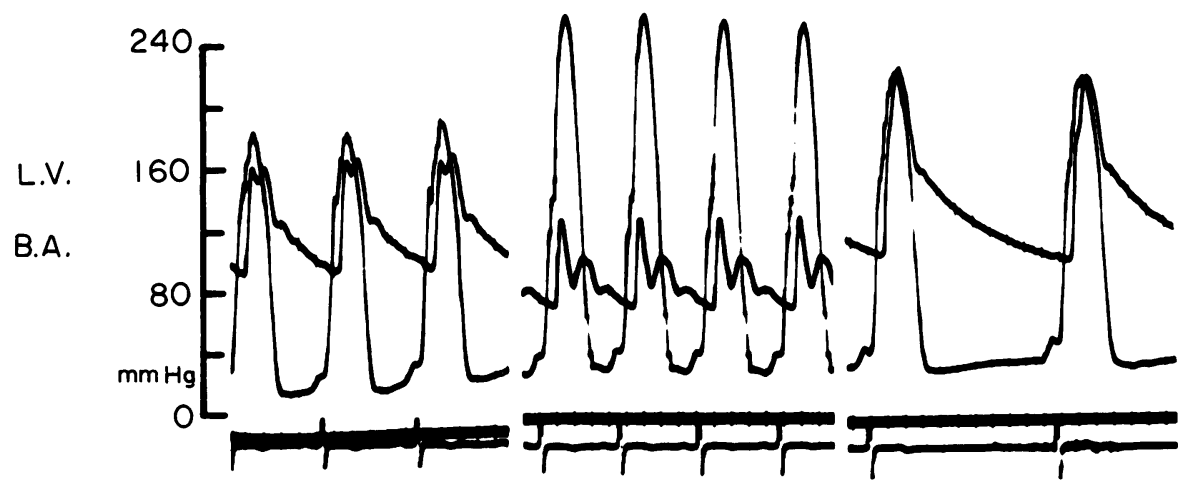

Fig. 8. Manipulation of the obstruction in HOCM (see text). The high LVEDP is noteworthy.

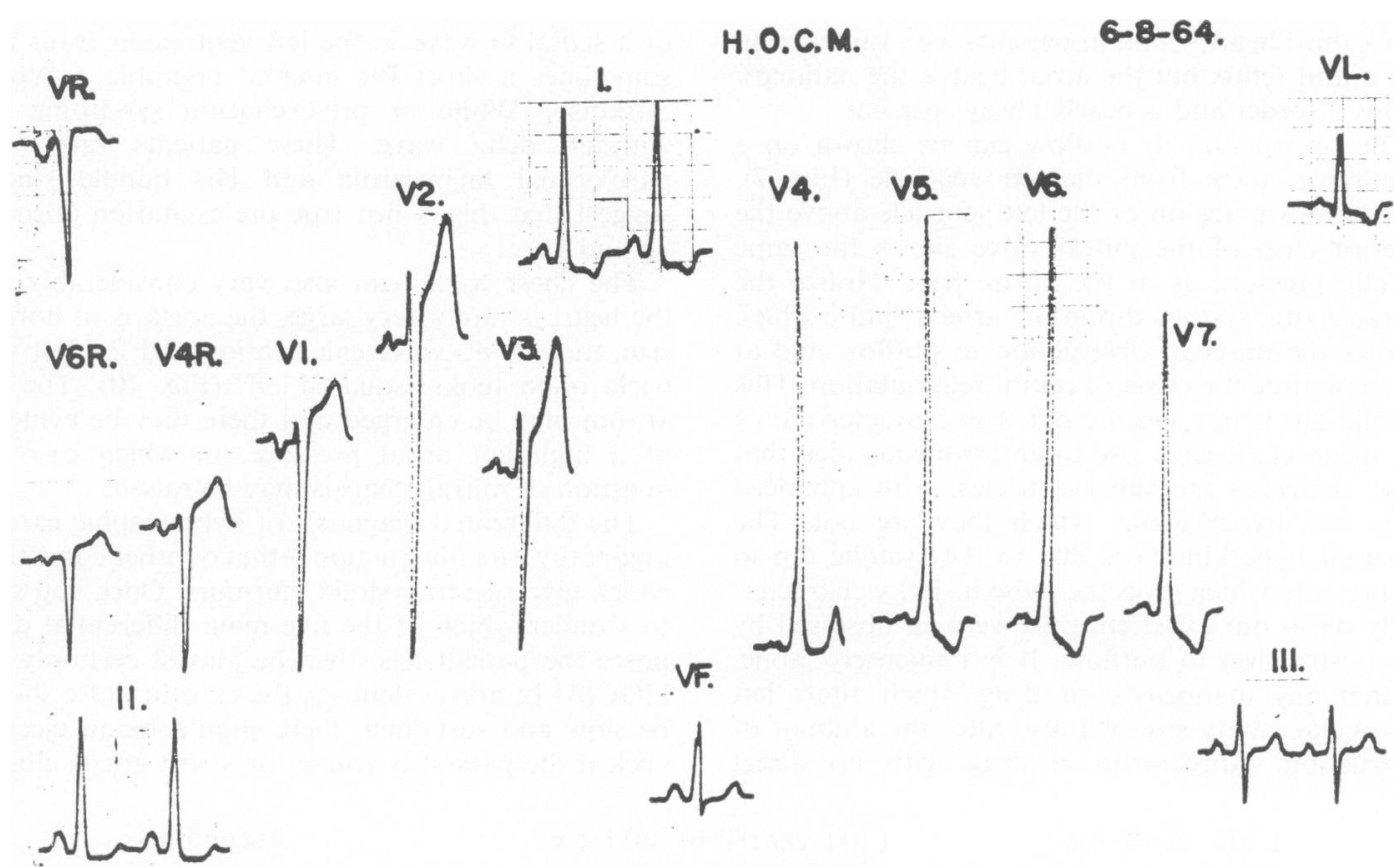

FIG. 9a. 'Typical' ECG in florid HOCM (see text), with pseudo-pre-excitation.

sound and valve calcification if the patient is old. There will probably be a dilated aorta. In discrete subaortic stenosis the patient is usually young and there is usually an early diastolic murmur which is never heard in uncomplicated hypertrophic cardiomyopathy. Incidentally, HOCM patients can get bacterial endocarditis, and infection of the mitral or aortic valves can modify the physical signs, but this is uncommon. The best differential diagnosis is undoubtedly non-rheumatic mitral regurgitation in which fading of the murmur after amylnitrite best distinguishes it from HOCM, prior to catheterization.

The start of the disease is not known. We believe that it is usually acquired after birth but that the predisposition is genetically based. We have shown 
Z. Y. A E t 27

C.N. 283635

A V R
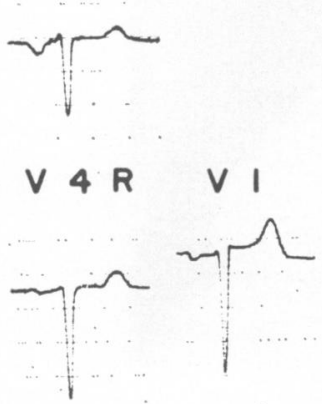

I I:
H. O.C.M.

$20 \cdot 10 \cdot 66$
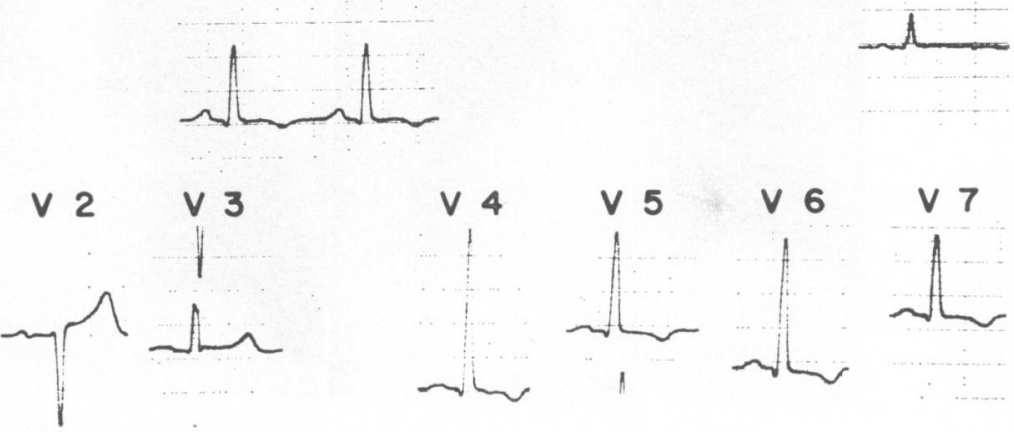

A V F

I I I.
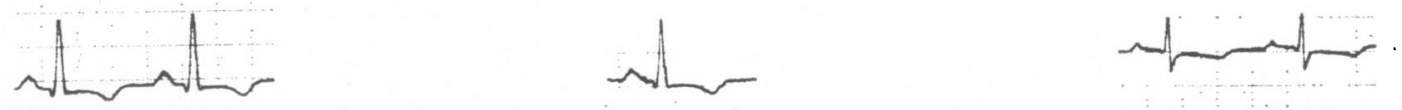

Fig. 9b. Less florid left ventricular hypertrophy in another patient.

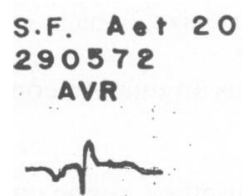

I.

19.5.66.

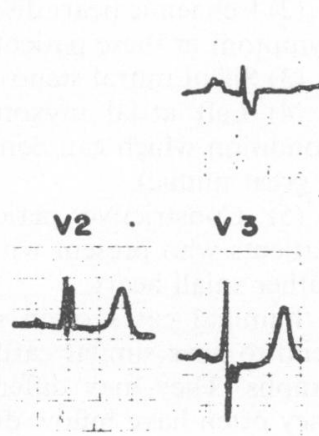

AVL

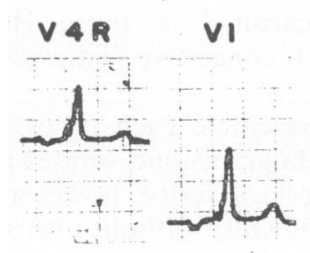

11.

III.
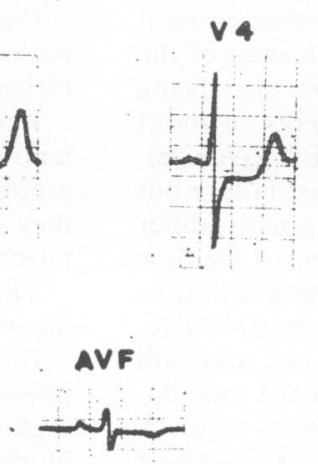

V 5

v 6
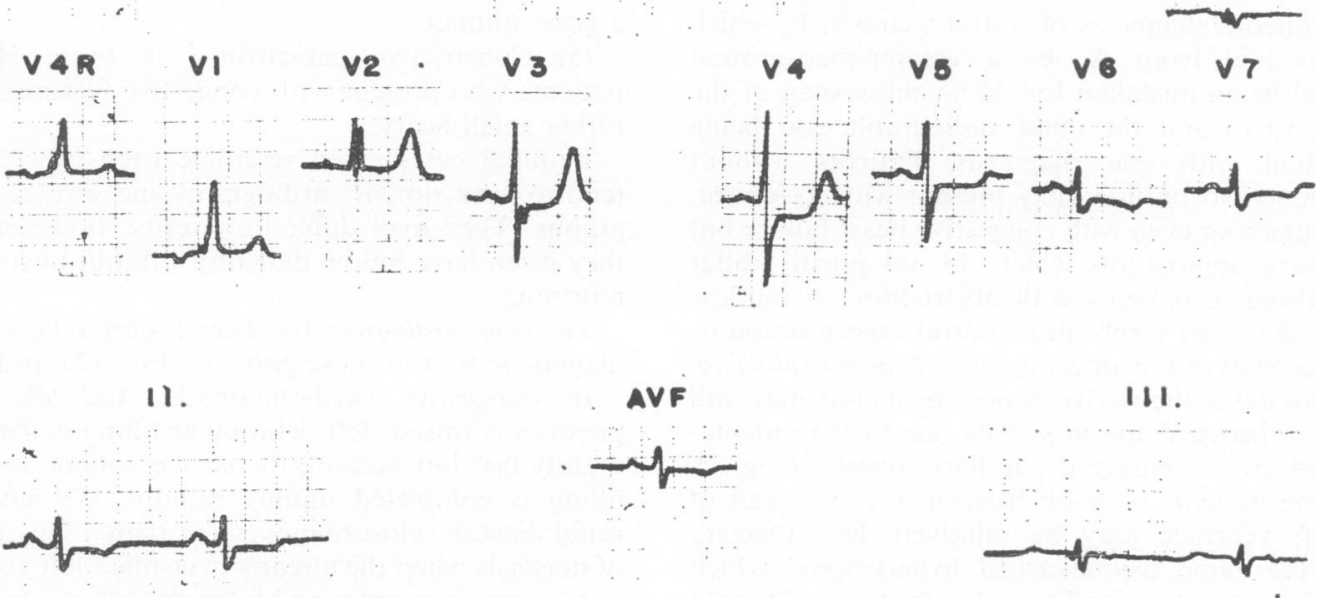

Fig. 9c. Right bundle branch block and widespread repolarization changes from another HOCM patient. 


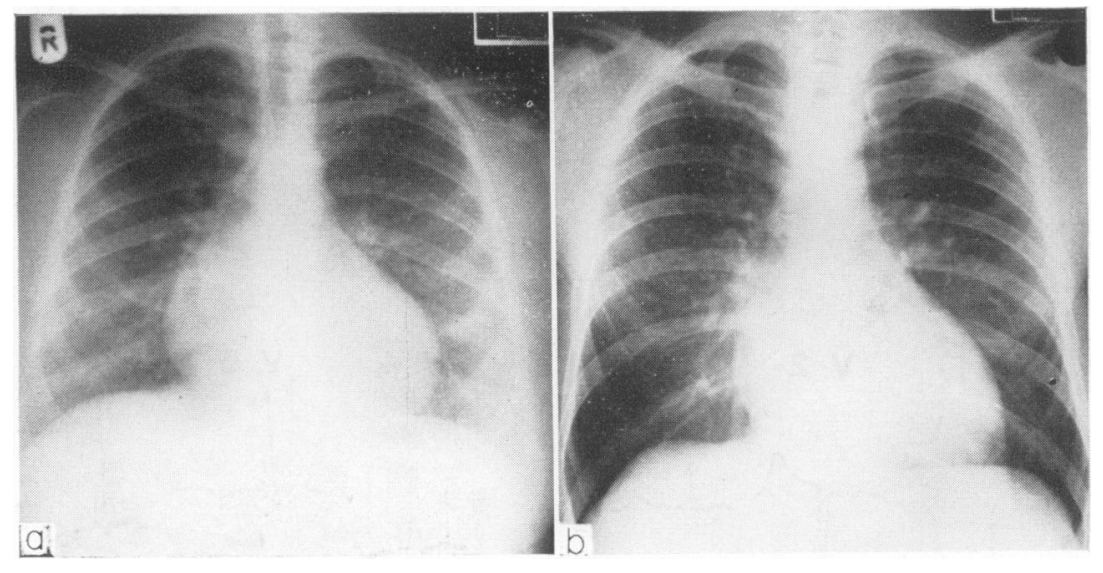

FIG. 10. (a) and (b) Representative plain chest radiographs in HOCM (see text).

that electrocardiographic conduction defects or other abnormalities are not present from birth. In Fig. 11 is an example with a left anterior hemi-block producing left axis deviation in the frontal plane and $Q$ waves in the septal leads mimicking infarction (Fig. 11b). This patient had had a normal cardiogram recorded 14 years earlier when his cardiac murmurs were first noted (Fig. 11a).

In patients without obstruction to outflow or in those who have lost it, murmurs are usually absent or non-specific but the atrial beat is predominant. There is often a widely split second heart sound because the left ventricular systolic interval is short, $A_{2}$ is early and $P_{2}$ may be slightly late if pulmonary hypertension is present. This has often given rise to an erroneous diagnosis of mitral stenosis, $\mathbf{P}_{\mathbf{2}}$ which is separated from $A_{2}$ by a greater-than-normal interval being mistaken for the opening snap of the mitral valve and the chest radiograph also being consistent with that diagnosis. Patients without obstruction to outflow may present with dyspnoea, with angina or even with congestive heart failure but they have angiograms which do not greatly differ from those of patients with obstruction to outflow except that they rarely show mitral regurgitation or displacement of the anterior cusp of the mitral valve. In these cases the cavity is not 'bent' but may still show a bizarre shape in systole due to the indentations of grossly enlarged papillary muscles (Fig. 4). In some patients with obstruction the free wall of the left ventricle may be relatively less affected, hence the name 'asymmetrical hypertrophy' which was given to this condition by Professor Donald Teare who first recognized it at necropsy. These patients without obstruction are much harder to diagnose unless one remembers the possibility of HOCM.

The differential diagnosis of HOCM withouto obstruction is from:

(1) COCM, if there are congestive features and because by this time the left atrium, the right ven-? tricle and the right atrium will all be dilated and give rise to some increase in heart size, which in rare instances may be considerable.

(2) Ischaemic heart disease, as angina is a common symptom in these patients.

(3) Silent mitral stenosis.

(4) Left atrial myxoma (another rogue cardiac condition which can compete with HOCM in being a great mimic).

(5) Constrictive pericarditis, in those HOCM patients who present with congestive features and a rather small heart.

Familial cases often resemble each other. They tend to have similar cardiograms and similar radiographs. They may differ in severity, however, and they often have inflow difficulty without obstructive murmurs.

The echocardiogram has been found to be a useful diagnostic tool in these patients (Fig. 12a and b).

In congestive cardiomyopathy the left atrial pressure is raised; left ventricular filling is therefore unduly fast but because the stroke volume is small, filling is completed unduly rapidly. We have this rapid diastolic closure movement then a long period of diastasis when the already over-filled left ventricle can accept no more blood from the left atrium. This should be contrasted with the echocardiographic 
(a) R.G. $28-7-54$.
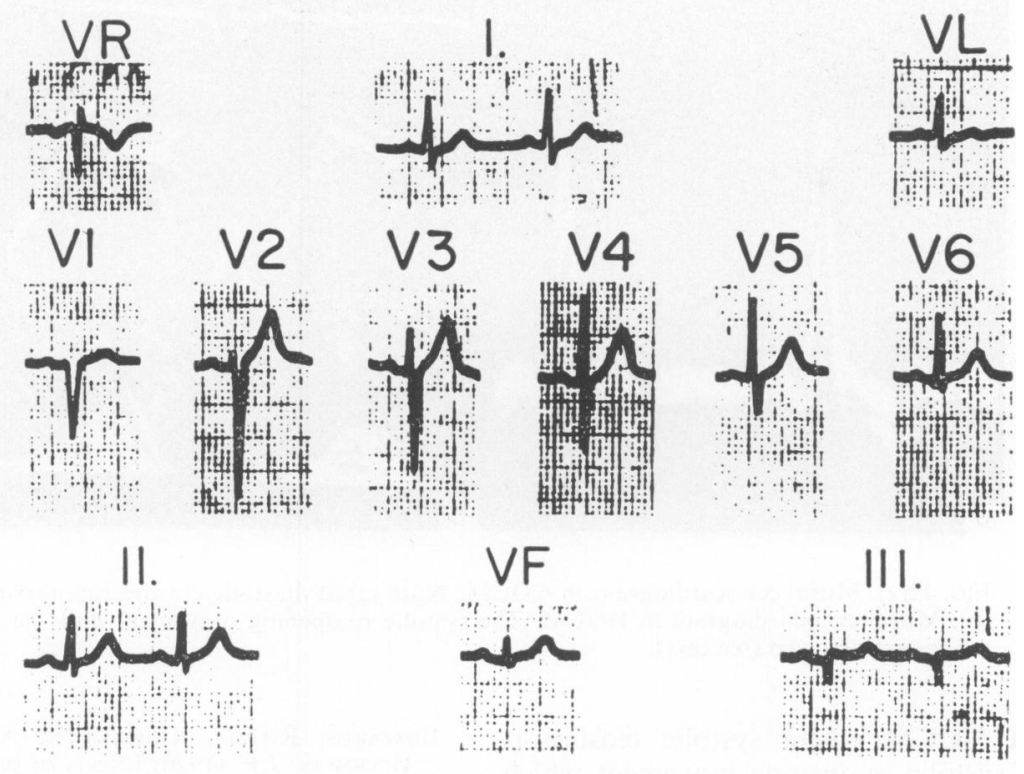

(b) R.G. 9-2-68.

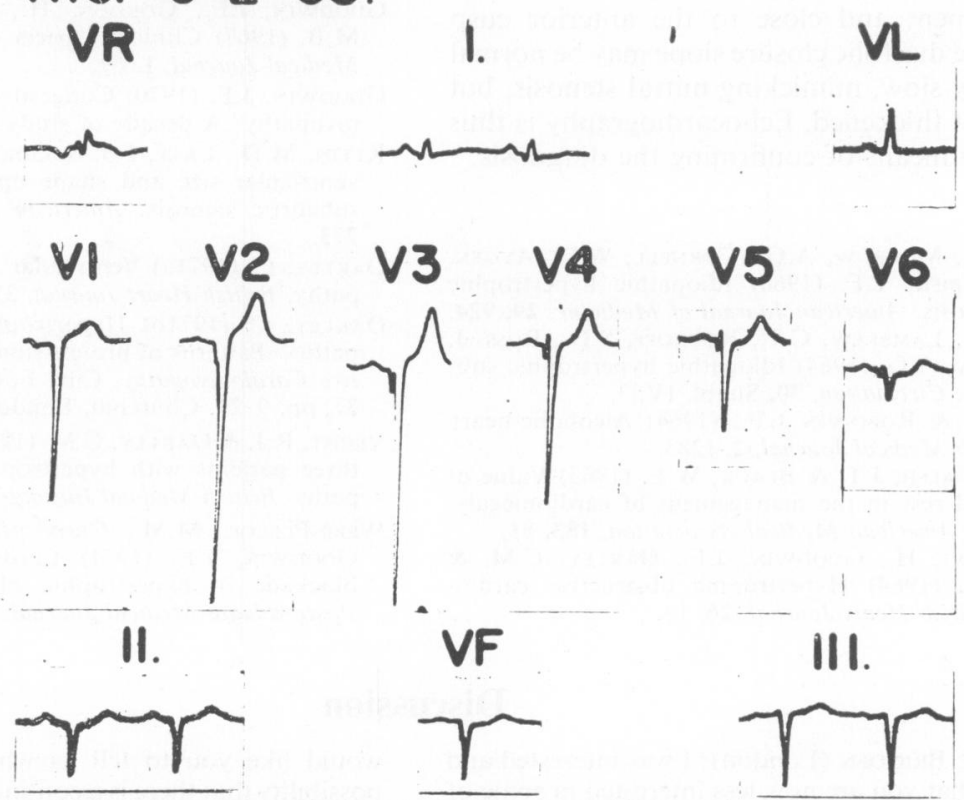

FIG. 11(a). Development of the ECG abnormality in HOCM (see text). (b) 14 years previously. 


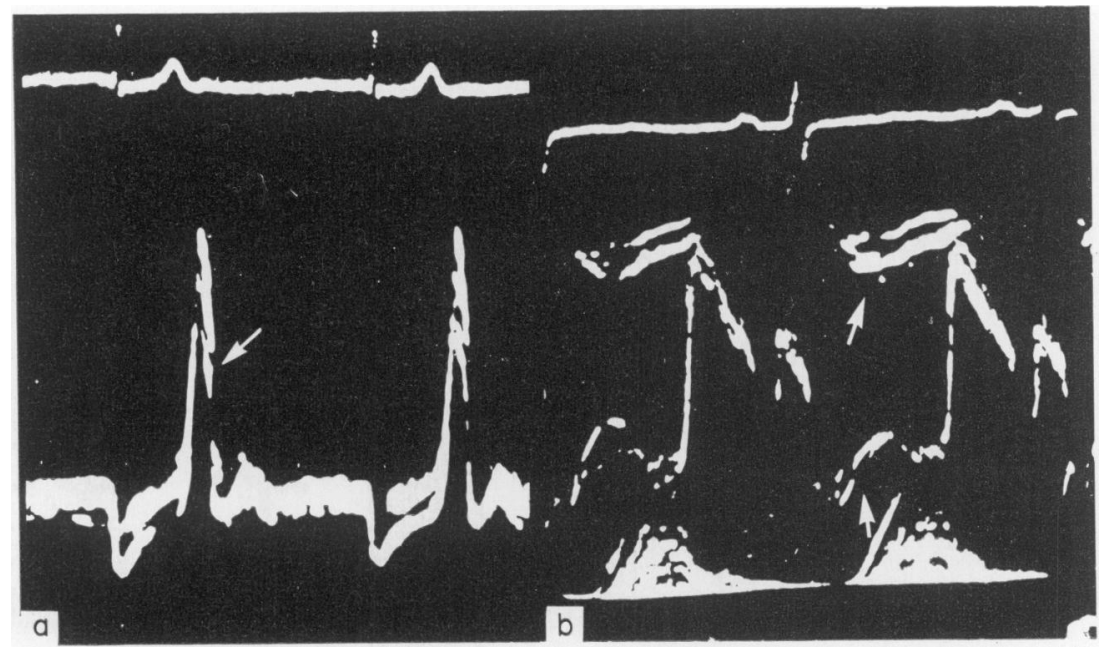

FIG. 12(a). Mitral echocardiogram in COCM. Note rapid diastolic closure rate (arrowed). (b). Mitral echocardiogram in HOCM. The systolic re-opening movement and the septal echoes are arrowed (see text).

appearance in HOCM where systolic closure is followed by a systolic re-opening movement which coincides with the anterior movement of the anterior cusp towards the septum, shown on the angiogram. This is virtually diagnostic of the obstructive form of the disorder. Secondly, the septal echoes are unduly prominent and close to the anterior cusp and thirdly, the diastolic closure slope may be normal or even unduly slow, mimicking mitral stenosis, but the valve is not thickened. Echocardiography is thus a non-invasive means of confirming the diagnosis.

\section{Bibliography}

Braunwald, E., Morrow, A.G., Cornell, W.P., Aygen, M.M. \& HiLBISH, T.F. (1960) Idiopathic hypertrophic subaortic stenosis. American Journal of Medicine, 29, 924.

Braunwald, E., Lambrew, C.T., Rockoff, S.D., Ross, J. JR \& Morrow, A.G. (1964) Idiopathic hypertrophic subaortic stenosis. Circulation, 30, Suppl. IV, 3.

Brigden, W.W. \& Robinson J. F., (1964) Alcoholic heart disease. British Medical Journal, 2, 1283.

BURCH, G.E., Walsh, J.T. \& BlaCK, W.L. (1963) Value of prolonged bed rest in the management of cardiomegaly. Journal of the American Medical Association, 183, 81.

Cohen, J., EfFat, H., Goodwin, J.F., OAKLey, C.M. \& STEINER, R.E. (1964) Hypertrophic obstructive cardiomyopathy. British Heart Journal, 26, 16.
EDWARDS, R.H.T., KristinsSon, A., Warrell, D.A. \& $\sigma$ GoODWIN, J.F. (1970) Effects of propranolol on response to exercise in hypertrophic obstructive cardiomyopathy. British Heart Journal, 32, 219.

Goodwin, J.F., Hollman, A., Cleland, W.P. \& Teare, D (1960) Cardiomyopathy simulating aortic stenosis. British Heart Journal, 22, 403.

Goodwin, J.F., Gordon, H., Hollman, A. \& Bishop M.B. (1967) Clinical aspects of cardiomyopathy. British Medical Journal, 1, 69.

GoodwiN, J.F. (1970) Congestive and hypertrophic cardiomyopathy: A decade of study. Lancet, i, 731.

Klein, M.D., LANE, F.J. \& Gorlin, R. (1965) Effect of left ventricular size and shape upon the haemodynamics of subaortic stenosis. American Journal of Cardiology, 15, 773.

OAKLEY, C. (1971a) Ventricular hypertrophy in cardiomyopathy. British Heart Journal, 33, Suppl. VI, 179.

OAKLEY, C. (1971b) Hypertrophic obstructive cardiomyopathy-Patterns of progression. In: Hypertrophic Obstructive Cardiomyopathy, Ciba Foundation Study Group No. 37, pp. 9-29. Churchill, London.

VeChT, R.J. \& OAKLEY, C.M. (1968) Infective endocarditis in three patients with hypertrophic obstructive cardiomyopathy. British Medical Journal, 1, 455.

Webb-Peploe, M.M., Croxson, R.S., Oakley, C.M. \& GooDwIN, J.F. (1971) Cardio-selective beta-adrenergic blockade in hypertrophic obstructive cardiomyopathy. Postgraduate Medical Journal, 47, Suppl. Jan. 93.

\section{Discussion}

DR WALlace BRIGden (London): I was interested and pleased to see that you are now less interested in some of the drugs that were very fashionable as diagnostic weapons a short time ago. I would like to ask Dr Oakley at this point whether she feels that there is a 'grey area'. I would like you to tell us whether you feel there is a possibility that there is a continuum rather than a bimodal position, i.e. hypertrophic cardiomyopathy on the one hand and your so-called congestive group on the other? Dr Celia OAKley: I am convinced that HOCM and 